\title{
The rat's simultaneous anticipation of remote events and current events can be sustained by event memories alone
}

\author{
E. J. CAPALDI and DANIEL J. MILLER \\ Purdue University, West Lafayette, Indiana
}

\begin{abstract}
After receiving events in a fixed order, A-B-C..., rats, like people, on being provided with A, may anticipate not only $\mathrm{B}$, a current anticipation, but also $\mathrm{C}$, a remote anticipation. In two experiments, we attempted to determine whether rats' remote anticipations are mediated by item cues (C elicited by $A$ ) or by position cues (C directly elicited by Position 3 cues, which generalize to Position 2). In Experiment 1, rats in a runway received two series of three trials, XNY and $\mathrm{ZNN}$, each in irregular order each day. $\mathrm{N}$ signified nonreinforcement; $\mathrm{X}, \mathrm{Y}$, and $\mathrm{Z}$ signified three qualitatively different food reinforcements. The rats manifested a remote anticipation by running faster on Trial 2 in the XNY series than in the ZNN series. Since the series were presented irregularly, Trial 2 performance cannot be explained on a positional basis alone. It can be explained on an item basis, by assuming that the memory of the Trial 1 reinforcer became associated not only with the Trial 2 event, but with the Trial 3 event as well. Thus on Trial 2 the memory of $X$ signaled $N$ and $Y$, whereas the memory of $Z$ signaled $N$ and $N$. Experiment 2 produced the same results, regardless of whether the XNY and ZNN series were presented in regular or irregular order. These results indicate that remote anticipations can be mediated by item associations. They offer no evidence that position associations can do the same, but they do not rule out that possibility.
\end{abstract}

Consider a serial-learning task in which each trial consists of the presentation of several independent events in a fixed order, A-B-C.... In the early trials, but not the later trials, of verbal serial learning, people provided with, for example, Event A often respond not with the next or adjacent event in the series, B (a current anticipation), but with some remote event of the series, for example, C (a remote anticipation; e.g., McGeoch \& Irion, 1952). A long-standing controversy in the field of human serial learning, and more recently in animal serial learning, is whether remote anticipations are due to interitem associations or position-item associations. As will become clear below, when a single list of items is presented, A-B-C..., item cues and position cues are simultaneously available to support anticipatory responding. Despite this confounding, and the fact that the position and item views are not mutually contradictory, it is not uncommon for individuals who favor one of these views to completely reject the other (see, e.g., Bower, 1981; Crowder, 1976; Slamecka, 1985).

The classical interitem view of Ebbinghaus (1885/1964) suggests that every item in the series becomes associated with every other item. Considering associations in the forward direction only, the theory suggests that associations are formed between adjacent items in the list (e.g., A $\rightarrow B$ ) and between remote items in the list (e.g., $A \rightarrow C$ ). Thus,

This research was supported in part by NSF Grant BNS-8515831 to E. J. Capaldi. Address correspondence to E. J. Capaldi, Department of Psychological Sciences, Purdue University, West Lafayette, IN 47907. when provided with Item $A$, the person anticipates not only Item $B$, but Item $C$ as well, responding, presumably, with the momentarily stronger associate. According to this view, remote anticipations arise from remote associations. The position-item view suggests that associations are formed between items and cues associated with the position of the item in the list. A variety of sources have been suggested for position cues. An obvious and frequently suggested source is time. Each item in the list occurs, within narrow limits, within a fixed time following the start of the list, and so each item could become associated with a distinctive temporal cue. In any case, remote anticipations have been ascribed to the stimulus generalization that occurs from later to earlier position cues in the list (see, e.g., Bower, 1981).

In a number of recent runway investigations employing rats, series have been constructed in which reinforcement and nonreinforcement have served as items. Each trip down the runway may be called a run. Runs may be reinforced or nonreinforced. Correct anticipation is indicated by greater speed on reinforced than on nonreinforced runs. That rats, like people, simultaneously anticipate current events and remote events was clearly indicated by the following findings, reported by Capaldi, Nawrocki, and Verry (1983; see also Capaldi, Nawrocki, Miller, \& Verry, 1986; Capaldi \& Verry, 1981; Capaldi, Verry, \& Nawrocki, 1982). Speeds on a target nonreinforced run were depressed, indicating correct anticipation of the current event, which was nonreinforcement. But speeds on the target nonreinforced run were elevated when the sub- 
sequent run was reinforced rather than nonreinforced, indicating that a remote anticipation was simultaneously active. In sum, running speed on a current run was the outcome of two distinct anticipations acting simultaneously-anticipation of the reinforcement event associated with the current run and that associated with a subsequent or remote run.

When a single list of items is employed (A-B-C...), item cues and position cues are simultaneously available to mediate remote associations; that is, the cues are confounded. A method of ensuring that animals were using item information was employed in the present experiments. It consisted of providing rats with two slightly different series of events. In Experiment 1 , in which the two-series procedure was used, a novel group called the interitem group was employed. The interitem group was novel because remote anticipations cannot be mediated in the absence of item information.

\section{EXPERIMENT 1}

In Capaldi et al.'s (1983) investigation, rats received two slightly different series of three runs each. The interrun interval was about $30 \mathrm{sec}$ and the interseries interval was about $15 \mathrm{~min}$. Runs terminated either in reinforcement ( $R$; .045-g Noyes food pellets) or in nonreinforcement $(\mathrm{N})$. One series was RNR (three successive runs terminated in $R, N$, and $R$, respectively) and the other was RNN. Each series occurred once each day, always in a given order-RNR first for half of the rats, RNN first for the other half. Speeds were eventually greater on all $\mathrm{R}$ runs than on all $\mathrm{N}$ runs, indicating correct anticipation of current events. But speeds were greater on Run 2 in the RNR series than in the RNN series, indicating that the reinforcement outcome on Run 3, a remote anticipation, was anticipated on Run 2. To explain current as well as remote anticipations in the investigation of Capaldi et al. (1983), two distinct sorts of assumptions are required. First, it must be assumed that the rats were able to determine on Run 2 which of the two series they were currently receiving. This determination must have been based on sensitivity to the regular order in which the series were presented each day, since Run 1 provided no information to this effect, terminating in $\mathbf{R}$ in both series. But although correct anticipatory responding could not have occurred in the absence of series order information, it could not be sustained by series order information alone. That is, the animal also had to determine which run of each series was currently occurring. To do this, series order information had to be combined with some other source of information. The other source of information was identified by Capaldi et al. (1983) as item information, and by Burns, Wiley, and Payne (1986) as position information (see also Burns \& Wiley, 1984; Burns, Wiley, \& Stephens, 1986).

In the present Experiment 1, as in the investigation of Capaldi et al. (1983), the rats received two series of three events each; however, the present series were modified in two important respects. The net result of these modifications was that remote anticipation on Run 2 and completely correct current anticipation on Run 3 could occur if the rats employed item associations, but could not occur on the basis of position associations alone. The first modification was that the two series were presented irregularly rather than regularly. The second modification was that three different types of R (.045-g Noyes pellets, Kellogg's Corn Pops, and Kellogg's Honey Smacks) were employed, rather than a single $R$. Call the three Rs $X$, $Y$, and $Z$, and represent one series as XNY, the other as ZNN. Assume, first of all, that the animal employs position information but not item information. On this basis, the animal would be able to determine that in both series, Positions 1, 2, and 3 received, respectively, consistent reinforcement, $0 \%$ reinforcement, and $50 \%$ irregular reinforcement. But neither on Run 2 nor on Run 3 would the animal know which of the two series was currently occurring, since the series were presented irregularly and item information was not utilized. Thus, an animal employing position cues and only position cues in connection with the XNY and ZNN series should run rapidly and nondifferentially on Run 1 (Position 1 cues, $100 \%$ reinforced), slowly and nondifferentialy on Run 2 (Position 2 cues, 0\% reinforced), and rapidly and nondifferentially on Run 3 (Position 3 cues, 50\% irregular reinforcement). In sum, if rats employed position cues and only position cues, there would be no indication on Run 2 of a remote anticipation, and on Run 3 running would not be slower to the $\mathrm{N}$ event than to the $\mathrm{Y}$ event.

If, however, the rats utilized item associations in connection with the XNY and ZNN series, they could, having received the first item of each series, anticipate the reinforcement outcome associated with each of the next two runs; that is, given Event $X$, the rats could anticipate runs terminating in $N$ and $Y$, and given Event $Z$, they could anticipate runs terminating in $N$ and $N$. Thus, rats given the XNY and ZNN series in irregular order could be expected to behave more or less as did the rats of Capaldi et al. (1983), which were given the RNR and RNN series in regular order. That is, they should run more rapidly on all $\mathrm{R}$ runs than on all $\mathrm{N}$ runs, and on Run 2 , they should run faster in the XNY series that in the ZNN series, a remote anticipation.

\section{Method}

Subjects. The subjects in Experiment 1 were 4 experimentally naive male albino rats purchased from the Holtzman Co., Madison, Wisconsin. They were about 92 days old at the start of the experiment.

Apparatus. The apparatus in Experiment 1 was a straight gray runway, $197.10 \mathrm{~cm}$ long, $10.1 \mathrm{~cm}$ wide, and enclosed by $13.85-$ $\mathrm{cm}$ sides; it was covered by a wire-mesh top on a hinged frame. The startbox and goalbox were $20.80 \mathrm{~cm}$ and $29.70 \mathrm{~cm}$ long, respectively, and were closed off by metal guillotine doors. Raising the startbox door started a completely silent 0.01 -sec digital clock, which was stopped when a photobeam located $158.13 \mathrm{~cm}$ beyond the startbox door and $7.50 \mathrm{~cm}$ in front of the goal cup was broken by the rat. Food (.045-g Noyes pellets, Kellogg's Corn Pops, or Honey Smacks) could be placed in the goal cup, which measured $4.00 \mathrm{~cm}$ 
in diameter and $1.50 \mathrm{~cm}$ in depth. When the photobeam was interrupted, an aluminum guillotine door was lowered, confining the rat to the goalbox.

Pretraining. On arrival at the laboratory, all rats were caged individually and were given ad-lib food and water for 17 days. They were then placed on deprivation, consisting of $14 \mathrm{~g}$ of Wayne Rodent Blox each day. On Days 1-6 of deprivation, each rat was handled for about $1 \mathrm{~min}$. On days 7-9, alley exploration was permitted for about $3 \mathrm{~min}$. On Day 7, six .045-g Noyes pellets were scattered in the alley; on Day 8, three Pops were so scattered; and on Day 9, three Smacks were so scattered. At about the 11/2-min mark of alley exploration each day, the guillotine doors were lowered and raised to acquaint the rat with this noise. Each rat received two series of three runs each in pretraining. Runs terminated in either six .045-g Noyes pellets, $15 \mathrm{sec}$ confinement in an unbaited goalbox, a single Smack, or approximately half of a Pop. Runs of a series were separated by about a 15-30-sec interval, series by about 10-15-min interval. One series may be symbolized as XNY, the other as ZNN. Y was always a Pop and the series terminating in $\mathrm{Y}$ is called the A series. The series terminating in $\mathrm{N}$ on Run 3 is called the B series. For 2 rats, $\mathrm{X}$ was six $.045-\mathrm{g}$ Noyes pellets and $\mathrm{Z}$ was a Smack. For the other 2 rats, the reverse was the case. On Day 10 of pretraining, each rat received its A series. On Day 11 , each rat received its B series. On Days 12 and 14, the A series was given followed by the B series, and on Day 13 the B series was given followed by the A series.

Experimental training. Experimental training began on Day 15 and lasted for 16 days. A run began with placement of the rat in the startbox; the startbox door was opened about $3 \mathrm{sec}$ later. After eating the reinforcer in the goalbox, the rat was placed in the interrun interval box, where water was available. The A and B series each occurred three times each day. A rat received all three runs of a series before the next rat was run. This procedure produced, as indicated, about a 15-30-sec interrun interval and a 10-15-min interseries interval. The 4 rats were run in irregular order over days. The order of presenting the series was ABBAAB on odd days and BAABBA on even days. If a rat had not entered the goalbox $60 \mathrm{sec}$ after the startbox door was raised, it was placed in the goalbox and a time score of $60 \mathrm{sec}$ was assigned. Following the last run of the day, the rats were returned to the home cage and were fed the daily ration about $10 \mathrm{~min}$ later. The food cup was baited noiselessly. Exposed food was kept outside the apparatus near the goalbox to provide a constant food odor.

\section{Results}

Figure 1 shows running speed on Run 1, Run 2, and Run 3 for each series, XNY and ZNN, in blocks of 2 days. The data are exceptionally clear. Running speeds for the two series did not differ on Run 1. With training, remote anticipation developed on Run 2, speed being greater on Run 2 in the XNY than in the ZNN series. Finally, on Run 3, correct current anticipation was manifested, running being faster on Run 3 in the XNY series than in the ZNN series. Each of these statements is supported statistically. An analysis of variance was performed over the data shown in Figure 1 (for this analysis, the data were broken down by days rather than by blocks of 2 days). The analysis revealed the following. Significant differences were associated with runs $[F(2,6)$ $=67.89, p<.001]$, with series $[F(1,3)=25.28$, $p<.05$ ], and, importantly, with the runs $\times$ series $\times$ days interaction $[F(14,42)=1.94, p<.05]$. A breakdown of this interaction, employing Newman-Keuls tests, revealed the following. In regard to current anticipation, on each of the last 2 days of training, speeds on every $\mathbf{N}$ run were significantly below that on any $\mathrm{R}$ run $(p<.05)$. In regard to remote anticipation, on each of the last 2 days of training, speed on Run 2 was greater in the XNY series than in the ZNN series ( $p s<.05$ ). That these differences developed with training is clear: On each of the first 2 days, for example, no difference between the series was significant on any run. On Run 1, no difference between the series was significant on any day. In support of what the posttests indicated about early training, an overall analysis over Days 1 and 2 revealed that no difference of any consequence even approached significance (runs, series, runs $\times$ series, runs $\times$ series $\times$ days).

\section{Discussion}

In Experiment 1, the rats correctly anticipated each current event, running faster on reinforced than on nonrein-

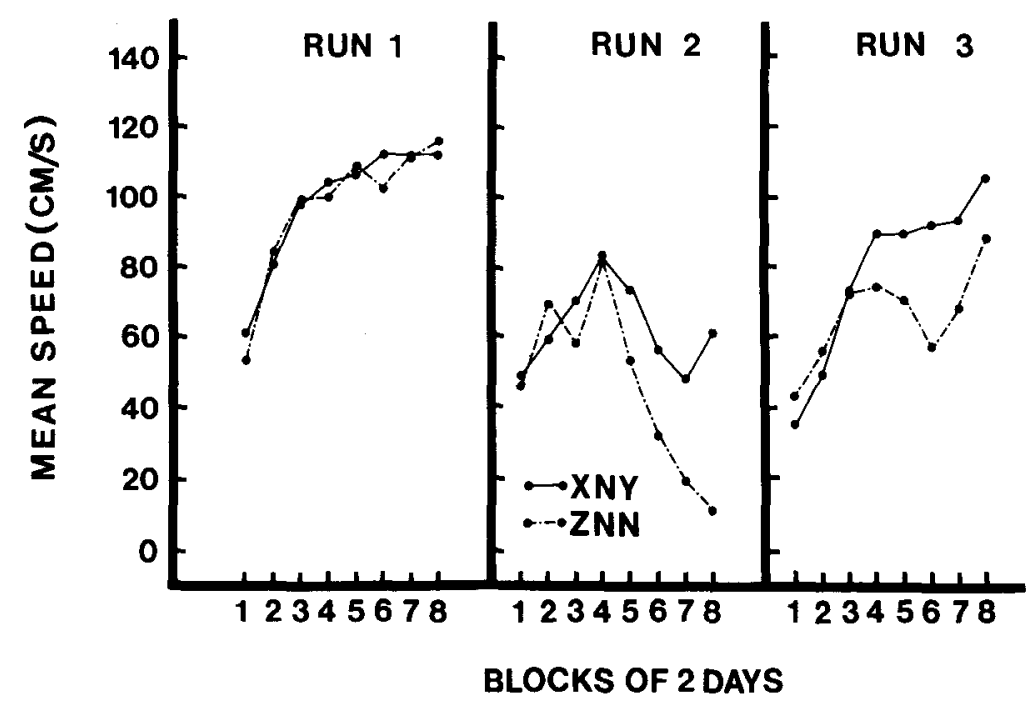

Figure 1. Running speed on Run 1, Run 2, and Run 3 of the XNY and ZNN series in blocks of 2 days. 
forced runs in each series. Too, the rats manifested a remote anticipation, running faster on Run 2 when Run 3 was anticipated to be reinforced (XNY series) than when Run 3 was anticipated to be nonreinforced (ZNN series). In Experiment 1, then, the rats were able to determine on Runs 2 and 3 which of the two series they were currently receiving and so could respond accordingly. What was the basis of this determination? Since the XNY and ZNN series were presented irregularly, the best the animal could do when employing position cues was to determine whether it was receiving Run 1, Run 2, or Run 3. It could not determine, however, which series it was receiving. Thus, the remote anticipation that occurred on Run 2 and the correct current anticipation of $\mathrm{Y}$ and $\mathrm{N}$ events on Run 3 cannot be explained by position cues. However, the findings of Experiment 1 are explicable in terms of item cues. This is because each series began with a distinctive item, $\mathrm{X}$ or $\mathrm{Z}$, which could be employed to anticipate the next two items of the series (XNY or ZNN).

Although the controversy regarding interitem and position-item associations is of relatively recent origin in animal serial learning (e.g., Burns, Wiley, \& Payne, 1986; Capaldi, Verry, Nawrocki, \& Miller, 1984; Haggbloom, 1985; Roitblat, Polage, \& Scopatz, 1983; Terrace, 1986), it has a long history in human serial learning (see, e.g., Crowder, 1976; Slamecka, 1985; Woodworth, 1938). Recognizing this, let us be absolutely clear about what was and was not shown by Experiment 1 . The results obtained in Experiment 1 demonstrate that cues associated with events of the series could be employed to correctly anticipate remote events and current events of the series and could not have been obtained in the absence of item information. This finding does not necessarily mean, however, that informative position cues, which were not provided in Experiment 1, would be incapable of mediating correct current and remote anticipations. Indeed, a considerable amount of additional information would have to become available before such an extreme conclusion could be adequately established. Experiment 2 represented a modest attempt to determine whether position cues contribute to the mediation of anticipatory responding in serial tasks.

\section{EXPERIMENT 2}

As indicated in conventional serial-learning investigations, item cues and position cues are simultaneously available to mediate anticipatory responding. Two groups were employed in Experiment 2, both of which received the two series XNY and ZNN. In both groups, each series occurred once each day. The difference between the groups was in the manner in which the series were presented-in regular order over days or in irregular order over days. Animals in Group C (for conventional) received the two series in a fixed order over days- $\mathrm{XNY}$ first each day for half the rats, ZNN first each day for the remaining half. Animals in Group I (for interitem only) received the two series in irregular order over days. The rats in Group I, like the rats in Experiment 1, could not show a correct remote anticipation on Run 2 and correct current anticipations on Run 3 in the absence of item cues. The rats in Group $\mathrm{C}$, however, had the option of employing position cues instead of or in addition to interitem cues; that is, we know, on the basis of results reported by Capaldi et al. (1983; see also Burns, Wiley, \& Payne, 1986), that when two different series are presented in a regular fixed order once each day, rats are capable of determining which of the two series they are currently receiving. Having made this determination, the rats can combine series information with item information (Capaldi et al., 1983), position information (Burns, Wiley, \& Payne, 1986), or both.

\section{Method}

Subjects. The subjects were 8 rats of the same description as those employed in Experiment 1.

Apparatus. The runway employed in Experiment 2 was slightly longer $(208.7 \mathrm{~cm})$ and slightly wider $(10.2 \mathrm{~cm})$ than that employed in Experiment 1, and was enclosed by higher sides $(22.72 \mathrm{~cm})$. The photobeam was located $175.27 \mathrm{~cm}$ beyond the startbox door and $7.5 \mathrm{~cm}$ in front of the goal cup, which was $4.2 \mathrm{~cm}$ in diameter and $1.85 \mathrm{~cm}$ deep.

Pretraining. Pretraining was similar to that employed in Experiment 1, except for the following differences. On Day 10, each rat received the X event of the A series. On Day 11, each rat received the three runs of its A series.

Experimental training. Experimental training was similar to that employed in Experiment 1, except for the following differences. Experimental training began on Day 12 of deprivation and lasted for 26 days. Each series occurred once each day. There were two groups of 4 rats each. In Group $C$, each rat received the two series in the same order each day-A first for 2 rats, A second for the other 2 rats. For 1 rat for which $A$ was the first series, $X$ was six .045 -g Noyes pellets; for the other, $X$ was a Smack. This was also the case for the 2 rats for which $A$ was the second series. Of course, when $\mathrm{X}$ was pellets, $\mathrm{Z}$ was a Smack, and vice versa. For Group 1, the $A$ and $B$ series occurred irregularly over days. For 2 rats, the order of the first series given on each of 8 successive days (repeated) was ABBABAAB. For the other 2 rats, the order was BAABABBA. For 1 rat in each condition, $X$ was six .045-g Noyes pellets; for the other, $X$ was a Smack. Of course, when $X$ was pellets, $Z$ was a Smack, and vice versa.

\section{Results}

Figure 2 shows running speed on Run 1, Run 2, and Run 3 for each series, XNY and ZNN, in blocks of 2 days, for Group I (top) and for Group C (bottom). On Run 1, neither group ran faster in one series than in the other. However, both groups showed remote anticipation on Run 2, running faster in the XNY series than in the ZNN series. This Run 2 difference appears on an absolute basis to be greater in Group C than in Group I. On Run 3, both groups showed correct current anticipation, running faster in the XNY than in the ZNN series. On an absolute basis, the Run 3 difference appears to be greater in Group $C$ than in Group I. On a statistical basis, however, no difference between the groups was significant on any run. Employing the data shown in Figure 2, we compared the groups on each run separately. On Run 1, only one difference of interest was significant, that due to blocks $[F(12,72)=34.81, p<.001]$. On Run 2, significant differences were associated with se- 
ries $[F(1,6)=5.91, p<.05]$ and the blocks $\times$ series interaction $[F(12,72)=11.84, p<.01]$, indicating remote anticipations on Run 2 . The tendency toward remote anticipation, however, was not differential with respect to groups: significant differences were not associated with either the groups $\times$ series interaction $[F(1,6)=1.15$, $p>.05]$ or the groups $\times$ series $\times$ blocks interaction $(F$ $<1)$. Of course, on Run 2 , the difference associated with blocks was significant $[F(12,72)=11.84, p<.01]$, and differences associated with groups approached but did not reach significance $[F(1,6)=4.56, .10>p>.05]$. On Run 3, significant differences were associated with series $[F(1,6)=23.37, p<.01]$ and blocks $\times$ series interaction $[F(12,72)=2.11, p<.05]$, indicating correct current anticipation on Run 3. This tendency was not differential with respect to groups, however; all of the following differences were nonsignificant: groups $[F(1,6)$ $=1.54, p>.05]$, groups $\times$ series $(F<1)$, and groups $\times$ series $\times$ blocks $(F<1)$. Differences due to blocks were highly significant $[F(12,72)=15.28, p<.001]$.

In a further effort to determine whether differences between the groups could be obtained, the last six blocks of each run were subjected to separate analyses. These analyses did not differ in any important respect from those described above. That is, no difference between the series on Run 2 or on Run 3 was affected by groups. Still another analysis-one that, like that in Experiment 1, employed all three runs-was performed. Again, there was no suggestion that the two groups differed statistically.

\section{Discussion}

In Experiment 2, Groups I and C correctly anticipated each current event, running faster on reinforced than on nonreinforced runs. Too, both groups manifested a remote anticipation, running faster on Run 2 when Run 3 was anticipated to be reinforced (XNY series) than when it was anticipated to be nonreinforced (ZNN series). On an absolute basis, the tendency toward a remote anticipation of Run 2 and appropriate current anticipation of $Y$ and $\mathrm{N}$ events on Run 3 was greater in Group $\mathrm{C}$ than in Group I. These differences, however, were not statistically significant.

Appropriate remote anticipations and certain correct current anticipations in Group I could not have been mediated in the absence of item cues. But in Group C, appropriate anticipations could have been mediated by item cues, position cues, or both. Had Group $\mathrm{C}$ shown better current and remote anticipation of events than did Group I, one plausible interpretation would be that position information, when available, can be combined with series information to augment anticipatory responding. However, since Group C failed to differ significantly from

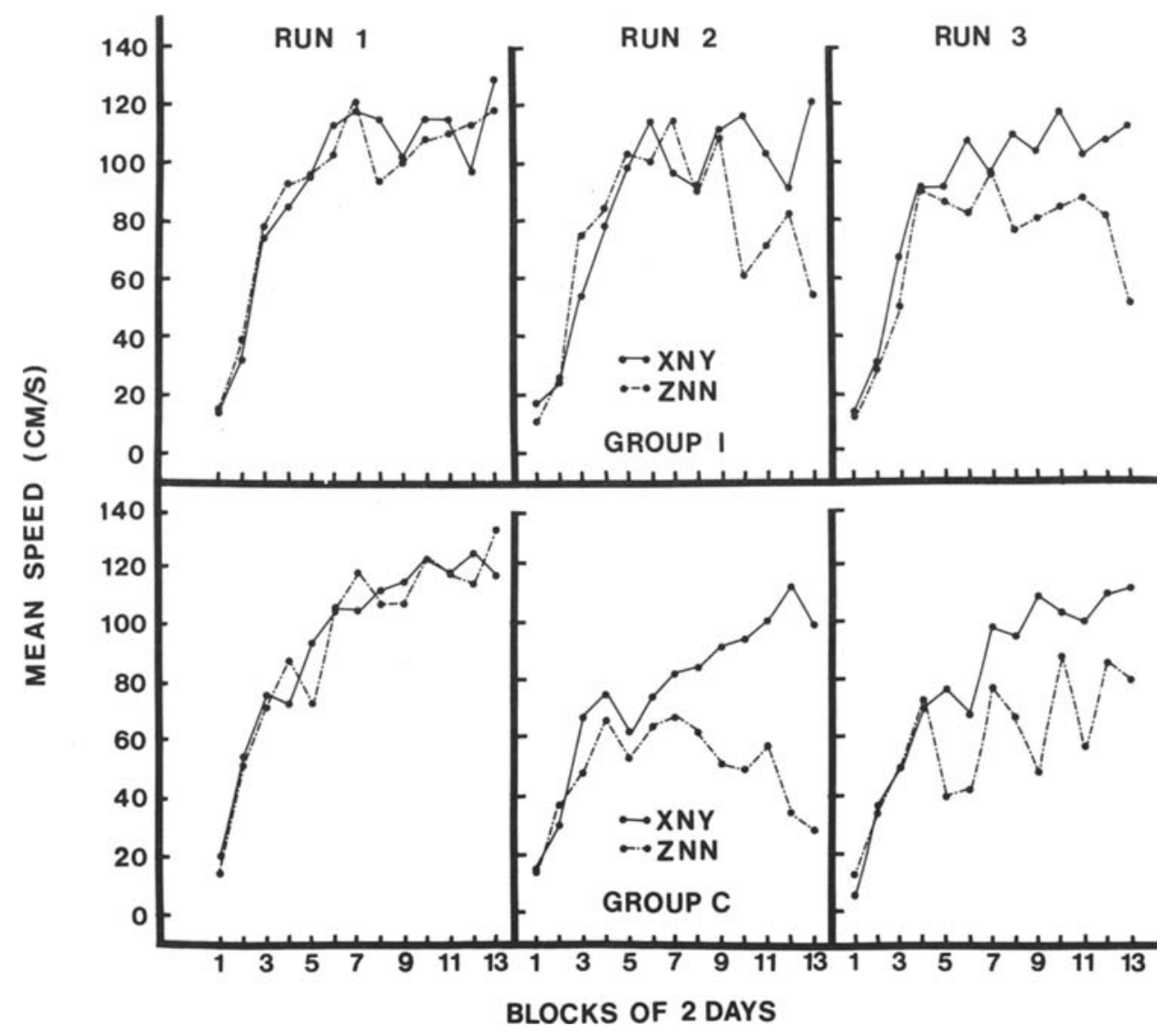

Figure 2. Running speed on Run 1, Run 2, and Run 3 of each series in blocks of 2 days for Group I (top) and Group C (bottom). 
Group I, we may say that in Experiment 2 no indication was provided that position information was utilized to anticipate events, although it may have been. We list three alternatives to the view that Groups I and C utilized only item information in Experiment 2. First, Group $\mathrm{C}$ may have utilized both position information and item information, with the additional position information failing to augment anticipatory responding beyond the level supported by item information alone in Group I. Second, Group $\mathrm{C}$ may have utilized position information instead of item information, with position information alone (Group C) failing to support better anticipatory responding than item information alone (Group I). The third possibility is perhaps the most interesting from a theoretical point of view. Although Group I could not have shown appropriate remote anticipations and certain current anticipations in the absence of item cues, once it did employ such cues it could then have utilized distinctive position cues as well; that is, position would be marked off with respect to the first item of the series (e.g., X plus Position 3 is Y). This view suggests that both Groups I and $\mathrm{C}$ may have employed item cues and position cues, and thus failed to differ. There are still other possibilities, but, whatever else may be the case, the present results are unique in that they strongly suggest that item information can be employed to mediate remote anticipations and current anticipations.

\section{GENERAL DISCUSSION}

Available, previously cited, findings clearly indicate that in an instrumental runway situation, rats are capable of anticipating simultaneously two distinct types of reinforcing events: those contingent on the current response (a current anticipation) and those contingent on sorne yetto-be-made response (a remote anticipation). These findings with animals are not unlike those obtained with people, who have been shown in serial tasks to anticipate remote as well as current events. Our major, but not exclusive, concern here was to identify the sort of information used to support remote anticipations. In available serial-learning investigations, with both people and animals, two distinct sources of information have been simultaneously available to mediate remote anticipations: item information and position information. Accordingly, in Experiments 1 and 2, we employed a novel group called the interitem group, in which two series of three runs each, XNY and ZNN, were presented to each rat in irregular order. As a consequence of irregular presentation of the series, remote anticipation on Run 2 (indicated by the rats' running faster on Run 2 in the XNY than in the ZNN series) and correct current anticipation on Run 3 (running faster to $\mathrm{Y}$ than to $\mathrm{N}$ ) could not occur in the absence of item information. Since the interitem group in both Experiment 1 and Experiment 2 showed correct remote anticipation on Run 2 and correct current anticipation on Run 3, we may conclude that item informatiaon can be employed to sustain both sorts of anticipations. Specifically, we assume that the memory of the Run 1 rein- forcer became associated not only with the Run 2 event but with the Run 3 event as well. Thus on Run 2 the memory of $X$ signaled $N$ and $Y$, whereas the memory of $\mathrm{Z}$ signaled $\mathrm{N}$ and $\mathrm{N}$.

Anticipation may link together a variety of learning situations normally thought of as unrelated. For example, it was recently suggested by Colotla and Bruner (1985) that sign tracking in the operant situation is an anticipatory phenomenon that has much in common with anticipation as it has been investigated in serial tasks by Capaldi et al. (1983). Moreover, it was recently suggested by Capaldi, Miller, and Nawrocki (1986) that serial learning and a family of tasks collectively referred to as working-memory tasks (see, e.g., Honig \& Dodd, 1986) are similar in important methodological respects. They also may be similar conceptually: anticipation has been emphasized in both. In one variety of working-memory task, delayed matching-to-sample, an initial or sample stimulus indicates which of two or more comparison or test stimuli should be selected, initial and test stimuli being separated by a retention interval. Retrospection implies passive memory of the sample stimulus in the retention interval. Retrospection has been the classical approach to retention in this situation. Prospection, lately emphasized, implies an anticipation of some aspect(s) of the trial outcome at the time the sample stimulus is presented. This analysis, generally speaking, is not unlike that provided here; for example, given Item A, which corresponds to the sample stimulus, the animal anticipates, for example, Item B, which corresponds to the test stimulus, A and B being separated by a retention interval. In considering the possible relationship between anticipation as it has been employed in the two types of tasks, the following comment seems in order. Prospection, as it has so far been examined in working-memory tasks, is similar to current anticipation in serial tasks in that it is some outcome of the current trial that is being anticipated. Whether, in working-memory tasks, remote events can be anticipated, and under what conditions, has yet to come under investigation. Another possible difference, which may turn out to be minor, has to do with the duration of the retention intervals thus far employed. In working-memory tasks, a retention interval of $60 \mathrm{sec}$ would be considered long. In recent serial-learning investigations, remote anticipations were formed without apparent difficulty when Items A and D were separated by $60 \mathrm{~min}$ and Items A and C were separated by 80 min (Capaldi, Miller, \& Nawrocki, 1986, Experiments 4 and 5); longer retention intervals have not been investigated. However, there is reason to believe that in working-memory tasks, long retention intervals may easily be bridged by employing appropriate experimental conditions (see, e.g., Capaldi, Nawrocki, Miller, \& Verry, 1986; Wright, Urcuioli, \& Sands, 1986).

Remote anticipation as investigated here consisted of anticipating, on a current run (Run 2), the reinforcing event contingent upon the immediately subsequent run (Run 3). Rats are capable of anticipating even more remote events. As one example, in Experiment 5 reported by Capaldi and Verry (1981), rats given Run 1 anticipated 
the reinforcing event associated with Run 5. Furthermore, in a series in which Run 5 was reinforced, Runs 2, 3, and 4 being nonreinforced, speeds increased systematically and progressively over Runs 2, 3, 4, and 5, until by Run 5 speed was very substantial, perhaps near maximum, as indicated by internal evidence (Run 1 speeds). Precise anticipatory responding of this sort is difficult to understand without assuming that rats possess a highly accurate representation of events even in relatively long series, so that on any run of the series, they are able to determine with considerable accuracy how many runs have already occurred, how many are yet to occur, and what reinforcing event is associated with each of the past and future runs. To do this, rats must employ both retrospection and prospection. It has recently been concluded that rats employ both retrospection and prospection in the radial maze (Brown \& Cook, 1986). Capaldi and Verry (1981) concluded that the ability of the rats to keep track of events in five-run series was accurate enough to suggest that the rats were counting items. Much evidence has since accumulated indicating that rats count and perhaps do so routinely (e.g., Capaldi \& Miller, in press; Capaldi, Nawrocki, \& Miller, 1986; Church \& Meck, 1984; Davis, 1986; Davis \& Albert, 1986). Thus rats, when forming remote anticipations, may be able to determine not only that some terminal run is to be reinforced, but also how many runs intervene between that terminal event and the current one. Counting, of course, is one way to keep track of the position of an item in a series. Indeed, according to one version of the position-item approach, items may become associated with some representation of their numerical position in the series (i.e., Position 1 is A, Position 2 is B, etc.; see Bower, 1981). We are suggesting that animals may count the items themselves, without necessarily ruling out the possibility that positions may be counted as well. In any event, the view that animals count items would explain a wide variety of rewardschedule data as well (e.g., Capaldi, 1966, 1967). Our view, outlined in greater detail elsewhere (Capaldi et al., 1984), is that reward-schedule data and serial-learning data are closely related and thus should be explained by the same set of principles.

\section{REFERENCES}

Bower, G. (1981). Theories of learning. Englewood Cliffs, NJ: Prentice-Hall.

Brown, M. F., \& CooK, R. G. (1986). Within-trial dynamics of radial arm maze performance in rats. Learning \& Motivation, 17, 190-205.

BURNs, R. A., \& WILEY, L. P. (1984). Interevent anticipation of liquid and solid sucrose rewards. Bulletin of the Psychonomic Society, 22, 571-573.

Burns, R. A., Wiley, L. P., \& Payne, T. L. (1986). Temporal cuing of runs in series of reward events reduces interevent anticipation. Animal Learning \& Behavior, 14, 190-196.

BURNS, R. A., WILEY, L. P., \& STEPHENS, J. (1986). Interevent anticipation with external cuing on runs and sucrose rewards. Psychological Record, 36, 101-107.

CaPaldi, E. J. (1966). Partial reinforcement: A hypothesis of sequential effects. Psychological Review, 73, 459-477.

CAPALDI, E. J. (1967). A sequential hypothesis of instrumental learning. In K. W. Spence \& J. T. Spence (Eds.), The psychology of learning and motivation (Vol. 1). New York: Academic Press.
Capaldi, E. J., \& Miller, D. J. (in press). Counting in rat: Its functional significance and the independent cognitive processes that constitute it. Journal of Experimental Psychology: Animal behavior processes.

Capaldi, E. J., Miller, D. J., \& Nawrocki, T. M. (1986). Retention interval and intertrial interval in a serial learning or delayed discrimination task. Journal of Experimental Psychology: Animal Behavior Processes, 12, 59-68.

Capaldi, E. J., Nawrocki, T. M., \& Miller, D. J. (1986, November). Counting and categorization in rats. Paper presented at the meeting of the Psychonomic Society, New Orleans, LA.

Capaldi, E. J., Nawrocki, T. M., Miller, D. J., \& Verry, D. R. (1986). Time between events as a retrieval cue: Recall and the temporal similarity between the storage and retrieval intervals. Journal of Experimental Psychology: Animal Behavior Processes, 12, 258-269.

Capaldi, E. J., Nawrocki, T. M., \& Verry, D. R. (1983). The nature of anticipation: An inter- and intraevent process. Animal Learming \& Behavior, 11, 193-198.

CAPALDI, E. J., \& VerRY, D. R. (1981). Serial order anticipation leaming in rats: Memory for multiple hedonic events and their order. Animal Learning \& Behavior, 9, 441-453

Capaldi, E. J., Verry, D. R., \& Nawrocki, T. M. (1982). Multiple hedonic memory: Memory for more than one hedonic event in the rat. Animal Learning \& Behavior, 10, 351-357.

Capaldi, E. J., Verry, D. R., Nawrockl, T. M., \& Miller, D. J (1984). Serial learning, interitem associations, phrasing cues, interference, overshadowing, chunking, memory, and extinction. Animal Learning \& Behavior, 12, 7-20.

Church, R. M., \& MECK, W. H. (1984). The numerical attribute of stimuli. In H. L. Roitblat, T. G. Bever, \& H. S. Terrace (Eds.), Animal cognition (pp. 445-464). Hillsdale, NJ: Erlbaum.

Colotla, V. A., \& Bruner, C. A. (1985). Sign-tracking as an antici pation process. In J. McGaugh (Ed.), Contemporary psychology: Biological processes and theoretical issues (pp. 145-157). Amsterdam Elsevier North-Holland.

Crowder, R. C. (1976). Principles of learning and memory. Hillsdale, NJ: Erlbaum.

DAvis, H. (1986, November). Self-imposed feeding restraint in the rat: A numerical discrimination. Paper presented at the meeting of the Psychonomic Society, New Orleans, LA.

Davis, H., \& AlberT, M. (1986). Numerical discrimination by rats using sequential auditory stimuli. Animal Learning \& Behavior, 14, 57-59.

EbBinghaus, H. E. (1964). Memory: A contribution to experimental psychology. New York: Dover. (Original work published 1885)

HaGGBLoOM, S. J. (1985). Serial learning and transfer in rats: Effects of changes in stimulus-stimulus associations, pattern structure, and serial position information. Animal Learning \& Behavior, 13, 370-374.

HoNIG, W. K., \& DoDD, P. W. D. (1986). Anticipation and intention in working memory. In D. F. Kendrick, M. E. Rilling, \& M. R. Denny (Eds.), Theories of animal memory (pp. 77-100). Hillsdale, NJ Erlbaum.

McGeoch, J. A., \& IRION, A. L. (1952). The psychology of learning New York: Longmans, Green and Co.

Roitblat, H. L., Polage, B., \& Scopatz, R. A. (1983). The representation of items in serial position. Animal Learning \& Behavior, 11 489-498.

SlameCKa, N. J. (1985). Ebbinghaus: Some associations. Journal of Experimental Psychology: Learning, Memory, \& Cognition. 11, 414-435.

TERRACE, H. S. (1986). A nonverbal organism's knowledge of ordinal position in a serial learning task. Journal of Experimental Psychology: Animal Behavior Processes, 12, 203-214.

WOODWORTH, R. S. (1938). Experimental psychology. New York: Holt. Wright, A. A., Urcuioli, P. J., \& Sands, S. R. (1986). Proactive interference in animal memory. In D. F. Kendrick, M. E. Rilling, \& M. R. Denny (Eds.) Theories of animal memory (pp. 101-125). Hillsdale, NJ: Erlbaum.

(Manuscript received February 9, 1987; revision accepted for publication June $25,1987$. 